\title{
Establishment of prognostic models for adenocarcinoma of oesophagogastric junction patients with neoadjuvant chemoradiotherapy: a real-world study
}

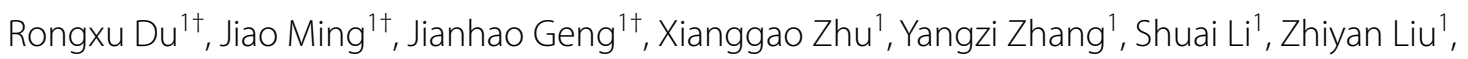
Hongzhi Wang ${ }^{1}$, Zhilong Wang ${ }^{2}$, Lei Tang ${ }^{2}$, Xiaotian Zhang ${ }^{3}$, Aiwen $\mathrm{Wu}^{4}$, Zhaode Bu${ }^{4}$, Yan Yan ${ }^{5}$, Zhongwu Li ${ }^{6}$, Yongheng $\mathrm{Li}^{\mathrm{i}^{*+}}\left(\mathbb{D}\right.$, Ziyu Li $\mathrm{i}^{4^{*+}}$ and Weihu Wang ${ }^{1 *+}$

\begin{abstract}
Background: Multimodal therapies based on surgical resection have been recommended for the treatment of adenocarcinoma of the oesophagogastric junction (AEG). We aimed to evaluate prognostic factors in AEG patients receiving neoadjuvant chemoradiotherapy and to build predictive models.

Methods: T3 - T4N + MO AEG patients with resectable Siewert type II/III tumours were enrolled in this study. All patients underwent neoadjuvant chemoradiation, followed by radical surgery or systemic therapy according to clinical response. Survival analysis was performed using the Kaplan-Meier method; multivariate analysis using the Cox proportional hazards method was also conducted. The Harrell concordance index (C-index) was used to test the prognostic value of models involving prognostic factors, and consistency between actual and predicted survival rates was evaluated by calibration curves.

Results: From February 2009 to February 2018, 79 patients were treated with neoadjuvant chemoradiotherapy; 60 patients of them underwent radical surgery. The R0 resection rate was $98.3 \%$, and $46.7 \%$ of patients achieved a major pathologic response (MPR), namely, a residual tumour issue less than $10 \%$. The 5 -year overall survival (OS) rate was $63 \%$, and the 5 -year progression-free survival (PFS) rate was $48 \%$. The incidence of grade 3 complications was $21.5 \%$, and no grade 4 complications were reported. According to the results of univariate and multivariate analyses, we
\end{abstract}

\footnotetext{
*Correspondence: yonghenglee@163.com; ziyu_li@hsc.pku.edu.cn; wangweihu88@163.com

${ }^{\dagger}$ Rongxu Du, Jiao Ming, Jianhao Geng contributed equally in this work and should be regarded as co-first authors.

${ }^{\dagger}$ Yongheng Li, Ziyu Li, Weihu Wang contributed equally in organizing this

study and should be regarded as co-corresponding authors.

${ }^{1}$ Key Laboratory of Carcinogenesis and Translational Research (Ministry

of Education/Beijing), Department of Radiation Oncology, Peking

University Cancer Hospital and Institute, Beijing 100142, People's Republic

of China

${ }^{4}$ Key Laboratory of Carcinogenesis and Translational Research (Ministry of Education/Beijing), Department of Gastrointestinal Surgery, Peking

University Cancer Hospital and Institute, Beijing 100142, People's Republic of China

Full list of author information is available at the end of the article
}

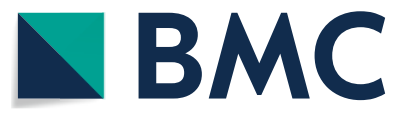

(c) The Author(s) 2022. Open Access This article is licensed under a Creative Commons Attribution 4.0 International License, which permits use, sharing, adaptation, distribution and reproduction in any medium or format, as long as you give appropriate credit to the original author(s) and the source, provide a link to the Creative Commons licence, and indicate if changes were made. The images or other third party material in this article are included in the article's Creative Commons licence, unless indicated otherwise in a credit line to the material. If material is not included in the article's Creative Commons licence and your intended use is not permitted by statutory regulation or exceeds the permitted use, you will need to obtain permission directly from the copyright holder. To view a copy of this licence, visit http://creativecommons.org/licenses/by/4.0/. The Creative Commons Public Domain Dedication waiver (http://creativecommons.org/publicdomain/zero/1.0/) applies to the data made available in this article, unless otherwise stated in a credit line to the data. 
included the neutrophil-lymphocyte ratio (NLR), prognostic nutrition index (PNI), eosinophilic granulocyte (EOS) and postoperative pathologic stage in nomogram analysis to establish prediction models for OS and PFS; the C-index of each model was 0.814 and 0.722 , respectively. Both the C-index and calibration curves generated to validate consistency between the actual and predicted survival indicated that the models were well calibrated and of good predictive value.

Conclusions: AEG patients achieved favourable downstaging and pathologic response after neoadjuvant chemoradiation, with acceptable adverse effects. Inflammation-based and nutrition-related factors and postoperative pathologic stage had a significant influence on OS and PFS, and the predictive value was verified through prognostic models.

Keywords: Adenocarcinoma of the oesophagogastric junction, Neoadjuvant chemoradiotherapy, Pathologic response, Inflammation-based and nutrition-related factors, Prediction models

\section{Background}

Oesophagogastric junction (EGJ) carcinoma is defined as carcinoma located $5 \mathrm{~cm}$ above and below the oesophagogastric junction. EGJ carcinoma ranks among the most common tumours worldwide and has become the most rapidly increasing tumour in Western countries [1-4]. Adenocarcinoma accounts for a great majority of EGJ carcinomas in East Asia [5-7]. The overall prognosis of this carcinoma is poor; although surgery is considered the fundamental treatment, local control and overall survival (OS) remain unsatisfactory, especially with advanced tumours [1]. Moreover, $70 \%$ of EGJ patients develop distant metastasis after primary tumour resection [8].

Given the deficiencies in single treatment, many studies have explored the value of comprehensive therapies for the treatment of AEG. Several prospective randomized control trials $[9,10]$ have indicated that neoadjuvant radiotherapy/chemoradiotherapy improves local control and survival among patients with potentially curable AEG. Yang et al. [11] compared preoperative and postoperative chemoradiotherapy in advanced gastric cancer, suggesting better OS, progression-free survival (PFS) and treatment compliance for preoperative chemoradiation. However, the application of neoadjuvant radiation or chemoradiation remains controversial. Some studies have suggested that chemoradiotherapy contributes to survival after surgery with limited lymph node dissection $[12,13]$, though not all patients can achieve optimal tumour regression in clinical practice; thus, selecting patients who may benefit from neoadjuvant chemoradiation is crucial for individualized treatment.

In addition, to provide more sufficient discussion about possible prognostic factors, several inflammation-based and nutrition-related factors, including the neutrophillymphocyte ratio (NLR), platelet-lymphocyte ratio (PLR), prognostic nutrition index (PNI), eosinophilic granulocyte (EOS) and fibrinogen (Fbg) [14-16], were explored in our study. Although some of these factors have been reported to have prognostic value for malignant tumours, e.g., a higher EOS value is suggested to be related to better survival in colon cancer [15], their application in AEG has rarely been reported. There are also predictive models involving inflammation-based and nutrition-related prognostic factors in oesophageal and gastric cancers [17, 18], but their conclusions are discordant, and other prognostic factors have rarely been included in these models.

Our study aimed to explore prognostic models, including clinical indices and treatment responses, and to select AEG patients who may benefit based on these models to promote individualized treatment.

\section{Methods \\ Patients}

Patients with previously untreated, biopsy-proven, locally advanced AEG were included in this retrospective study. Detailed inclusion criteria were as follows: patients (1) aged 18-75 years old; (2) diagnosis of oesophagogastric junction adenocarcinomas by endoscopy and biopsy pathology; (3) primary tumours of T3-T4 stage and positive lymph nodes; (4) primary tumours considered to be resectable before start of treatment; (5) Siewert II or III type of AEG, according to the definition from Siewert and Stein [19], whereby type II is defined as a tumour invading the EGJ, in which the centre is located between $1 \mathrm{~cm}$ above and $2 \mathrm{~cm}$ below the EGJ, and type III is defined as a tumour invading the EGJ, in which the centre is located $2 \mathrm{~cm}-5 \mathrm{~cm}$ below the EGJ; (6) Eastern Cooperative Oncology Group (ECOG) score 0 or 1 before start of treatment, with tolerance to chemoradiation; and (7) neutrophil count greater than 1,500 cells $/ \mu \mathrm{L}$, haemoglobin greater than $100 \mathrm{~g} / \mathrm{L}$, and platelet count greater than $100,000 / \mu \mathrm{L}$.

All patients underwent imaging staging and peritoneal washings before the start of treatment. Patients with distant metastases suggested by imaging examination or peritoneal washings were excluded. 


\section{Haematological index measurement}

Baseline blood data were obtained by collecting blood from the peripheral vein of each patient within 1 week before neoadjuvant chemoradiation. The EOS and Fbg were obtained directly by the blood test, and the NLR and PLR were defined as the absolute neutrophil count and platelet count divided by the absolute lymphocyte count, respectively. The PNI was calculated using the following formula: $10 \times$ serum albumin $(\mathrm{g} / \mathrm{dL})+0.005 \times$ total lymphocyte count (per $\mathrm{mm}^{3}$ ) [20]. The X-tile program was used to determine the optimal cut-off value of the above factors for predicting prognosis [21].

\section{Assessment}

The patients were diagnosed with AEG through endoscopy and biopsy. Endoscopy, endoscopic ultrasonography and computer tomography (CT) were used to determine the location and Siewert type of primary tumours and clinical stage before the start of treatment, for the patients with staging in question, discussion with radiographers would be carried out for more accurate clinical stage. After completion of neoadjuvant chemoradiation, the patients were subjected to clinical response assessment by $\mathrm{CT}$ to evaluate the therapeutic effect based on RECIST version 1.1 [22] and determine subsequent treatment. We used the pathological analysis results after surgery as the standard criteria to evaluate the R0 resection rate and pathologic response rate. Patients with less than $10 \%$ residual tumour tissue in relation to the macroscopically identifiable tumour bed of the primary tumour site were considered as having a major pathologic response (MPR) [23]. The tumours were staged clinically and pathologically according to the AJCC 8th edition of cTNM stage and ypTNM stage, respectively.

\section{Treatment}

All the patients gave their written informed consent before any treatments, including radiotherpy, chemotherapy and surgery. All patients enrolled in this study accepted neoadjuvant chemoradiation. Volumetric-modulated Arc Therapy (VMAT) was executed in this study. Cone beam CT (CBCT) was used to verify the planned target volume with actual location of tumors during radiotherapy process, in the first three times of radiation, and once a week from the 2nd week of radiotherapy. The gross tumour volume (GTV) included primary tumour and metastatic lymph nodes; the clinical tumour volume (CTV) included the GTV and high-risk lymphatic drainage area. External beam radiotherapy was applied to the CTV at a total dose of 45 Gy in 25 fractions of 1.8 Gy and the GTV at a total dose of $50 \mathrm{~Gy}$ in 25 fractions of $2.0 \mathrm{~Gy}, 5$ times a week. An example of realistic dose distribution and Dose-Volume Histogram was shown in Fig. 1. Patients accepted concurrent chemotherapy during radiation, and the standard regimen in this study was oxaliplatin (intravenous drip, $40 \mathrm{mg} / \mathrm{m}^{2}, \mathrm{~d} 1$ ) combined with S-1 (oral administration, $30 \mathrm{mg} / \mathrm{m}^{2}$, bid, d1-d5) weekly since March 2014, before when 17 patients orally took S-1 (25-35 mg/m², bid, d1-d5) weekly.

For those with newly distant metastases suggested by $\mathrm{CT}$ evaluation or surgery findings, systemic treatment should be taken into consideration, including systemic chemotherapy with or without local radiotherapy or cytoreductive surgery. If the response evaluation indicates a stable disease (SD), partial response (PR) or complete response (CR) status, according to RECIST v1.1, radical surgery would be taken into consideration, with the standard surgical method being total gastrectomy with D1+/D2 lymphadenectomy.

\section{Statistical analysis}

Statistical analysis was carried out with SPSS software, version 24.0 (SPSS Inc., Chicago, IL, USA). OS was defined as the time from diagnosis to date of death from any cause or last follow-up, and PFS was calculated from the start of chemoradiation to initial progress, death from any cause or last follow-up without progress. Survival analysis was performed with the Kaplan-Meier method, and the results were compared by the log-rank test. Multivariate analysis of independent risk factors for survival was performed with the Cox proportional hazards method, and logistic regression analysis was conducted for risk factors related to recurrence and metastasis. We used Fisher's exact test to clarify the relationship between different factors. All variables with significant results by univariate analysis $(\mathrm{P}<0.05)$ were included in the multivariate model. ROC curves were also produced for blood markers to assess their ability to predict prognosis. The Harrell concordance index (C-index) was employed to test the prognostic value of the model involving all the above prognostic factors, and consistency between the actual observed survival rates and predicted survival rates was evaluated by calibration curves.

\section{Results}

\section{Patients and treatments}

From February 2009 to February 2018, we included 79 AEG patients who accepted treatment at Beijing Cancer Hospital. All patients accepted chemoradiation as their first therapy; the median age was 63 years old. The major initial symptoms of these patients were dysphagia and abdominal pain. Seventy-four patients completed the whole radiation course without pause or delay; the other 5 patients failed to complete the radiotherpy continuously due to treatment-related complications. The 

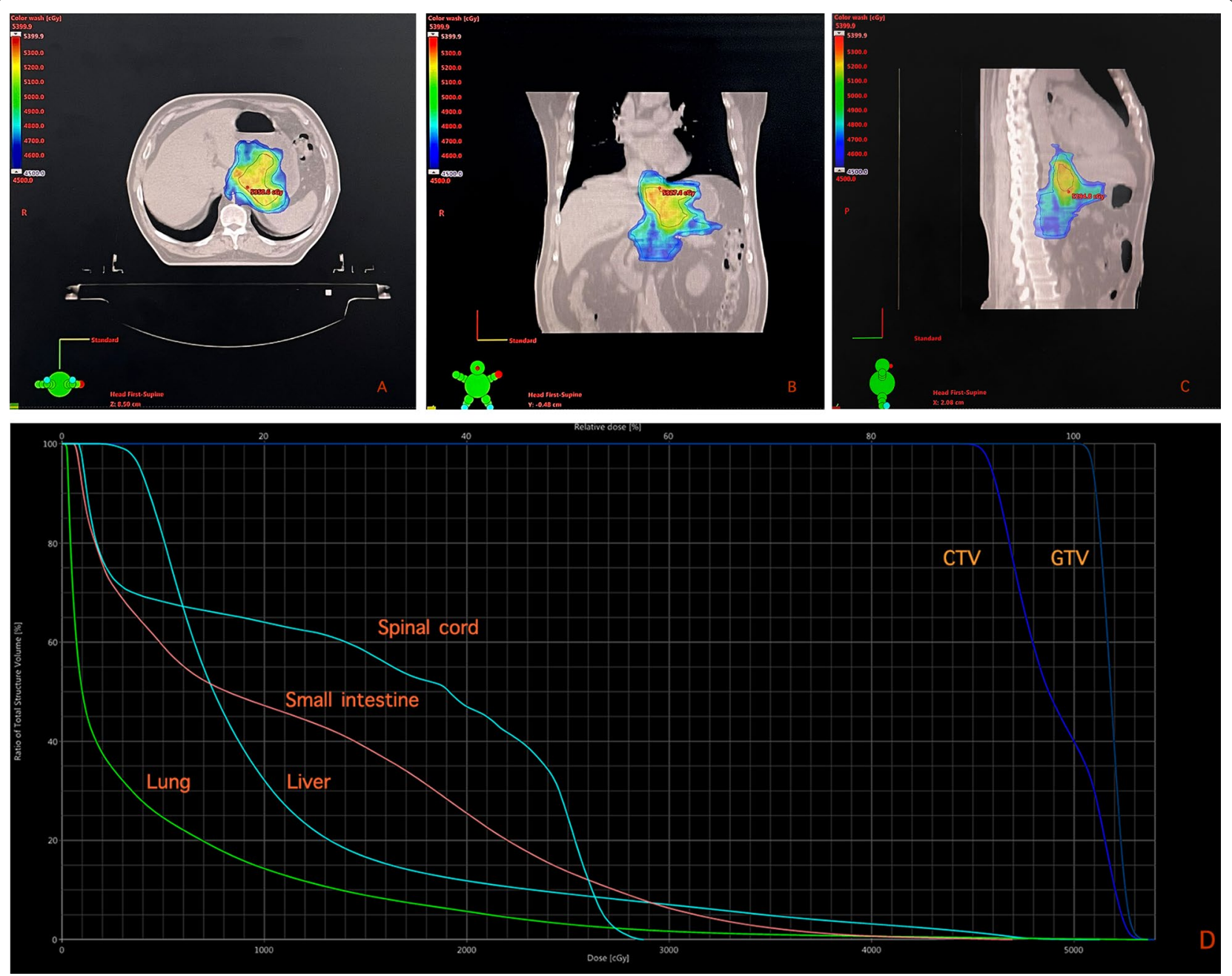

Fig. 1 The color wash mapping depicting the radiation dose distribution of a realistic case in the transverse section (A), coronal section (B), and sagittal section (C). The Dose-Volume Histogram (DVH) indicated the dose received by normal tissues (lung, liver, small intestine and spinal cord) and target volumes (D)

delay or deficiency of courses was no more than 5 fractions. Ten patients did not accept further treatment after chemoradiation due to personal reasons, and 9 accepted systemic treatment because of distant metastases found in the evaluation after chemoradiation. The details of the patients' clinical factors are shown in Table 1. From the survival analysis involved the characteristics in this table, we could tell that age, sex, ECOG score, Siewert type, Lauren type, and clinical $\mathrm{T} / \mathrm{N}$ stage didn't have significant influence on OS or PFS of patients in this study.

Ultimately, 60 patients underwent radical surgery: open total gastrectomy (41) and laparoscopic-assisted total gastrectomy (19) with D1+/D2 lymphadenectomy. Only one of the patients had a positive margin, and the R0 resection rate was $98.3 \%$. Postoperative pathological analysis showed that 8 patients (13.3\%) achieved a pathologic complete response (pCR) and that $28(46.7 \%)$ achieved MPR. The number of lymph nodes surgically removed was $23.14 \pm 8.67$, the number of positive lymph nodes was $0.57 \pm 0.83$, and 20 patients (33.3\%) were diagnosed with local lymph node metastasis. Fifty-five patients (91.7\% of patients treated with surgery, $69.6 \%$ of all patients) achieved tumour downstaging confirmed by pathological examination. Fifty-nine patients $(74.7 \%$ of all patients) achieved tumour downstaging confirmed by pathological or imaging examinations. The details of the surgery and surgical results are listed in Table 2. Postoperative pathologic stage was shown to be a significant prognostic factor both in OS $(\mathrm{P}<0.001)$ and PFS $(\mathrm{P}<0.001)$, other variables like surgery method, scope, Tumour Regression Grade (TRG) and postoperative condition of lymph nodes failed to affect the survival of patients significantly. 
Table 1 Overview and univariate analysis of the clinical characteristics of the patient cohort

\begin{tabular}{|c|c|c|c|}
\hline Patient characteristics & $\mathrm{N}(\%)(\mathrm{N}=79)$ & $\begin{array}{l}\text { Log-rank } \\
\text { P value } \\
\text { (with OS) }\end{array}$ & $\begin{array}{l}\text { Log-rank } \\
\text { P value } \\
\text { (with } \\
\text { PFS) }\end{array}$ \\
\hline Age & $\begin{array}{l}\text { 44-74 years } \\
\text { (median age } \\
63 \text { years) }\end{array}$ & - & - \\
\hline Sex & & 0.182 & 0.229 \\
\hline Male & $76(96.2 \%)$ & & \\
\hline Female & $3(3.8 \%)$ & & \\
\hline ECOG score & & 0.830 & 0.517 \\
\hline 0 & $66(83.5 \%)$ & & \\
\hline 1 & 13(16.5\%) & & \\
\hline Siewert type & & 0.078 & 0.120 \\
\hline Siewert II & $44(55.7 \%)$ & & \\
\hline Siewert III & 23(29.1\%) & & \\
\hline Unavailable & $12(15.2 \%)$ & & \\
\hline Lauren type & & 0.652 & 0.787 \\
\hline Intestinal type & $39(49.4 \%)$ & & \\
\hline Diffuse type & $14(17.7 \%)$ & & \\
\hline Mixed type & 15(19.0\%) & & \\
\hline Undefined & $11(13.9 \%)$ & & \\
\hline Clinical T stage & & 0.301 & 0.999 \\
\hline T3 & $16(20.3 \%)$ & & \\
\hline T4 & $63(79.7 \%)$ & & \\
\hline Clinical N stage & & 0.450 & 0.962 \\
\hline N1 & $19(24.05 \%)$ & & \\
\hline N2 & $41(51.9 \%)$ & & \\
\hline N3 & $19(24.05 \%)$ & & \\
\hline
\end{tabular}

ECOG: eastern cooperative oncology group

\section{Survival}

The median follow-up time was 38.5 months (5-106 months). There were 6 cases of local recurrences and 27 of distant metastases during follow-up. At the end of follow-up, 31 patients had died due to the cancer, 1 patient died due to postoperative complications, and 2 patients died due to other acute diseases. The 5 -year OS rate was 63\%; the 5-year PFS rate was $48 \%$. The median OS was 85 months, and the median PFS was 49.94 months.

\section{Complications}

Some patients experienced chemoradiation-related toxicity. Seventeen patients $(21.5 \%)$ had grade 3 toxicities after chemoradiation, but no grade 4 toxicity occurred in this study. Nine patients $(11.4 \%)$ had grade 3 haematologic toxicities, which ranked as major complication in this study; five patients $(6.3 \%)$ had grade 3 radiation oesophagitis and one patient (1.3\%) had grade 3 radiation pneumonitis; moreover, three
Table 2 Overview and univariate analysis of surgical data and surgery-related outcomes

\begin{tabular}{llll}
\hline Surgery-related factors & $\mathbf{N}(\%) \mathbf{( N = 6 0 )}$ & $\begin{array}{l}\text { Log- } \\
\text { rank P } \\
\text { value } \\
\text { (with } \\
\text { OS) }\end{array}$ & $\begin{array}{l}\text { Log-rank } \\
\text { P value } \\
\text { (with }\end{array}$ \\
PFS)
\end{tabular}

patients with grade 2 radiation pneumonitis got postsurgical pulmonary infection or difficulty in weaning, resulting in prolonged hospital stay after surgery. Altogether, seventeen patients (28.3\%) experienced postoperative complications needing intervention, and one patient ( $1.7 \%$ of patients who underwent surgery) died of massive haemorrhage in the hospital 2 weeks after surgery.

\section{Inflammation-based and nutrition-related factors}

As inflammation-based and nutrition-related factors were considered to be possible prognostic factors in our analysis, we used the X-tile program to determine the cut-off values of NLR, PLR, EOS, PNI and Fbg before we included them in survival analysis, and the results were $2.2,169.7,0.1,55.9$ and 345.5, respectively. The cut-off value and corresponding analysis of these factors are 
Table 3 Inflammation-based and nutrition-related factors involved in the analysis

\begin{tabular}{llll}
\hline Patient characteristics & $\mathbf{N}(\%)(\mathbf{N}=\mathbf{7 9})$ & $\begin{array}{l}\text { Log-rank P } \\
\text { value } \\
\text { (with OS) }\end{array}$ & $\begin{array}{l}\text { Log-rank } \\
\text { P value } \\
\text { (with } \\
\mathbf{P F S})\end{array}$ \\
\hline $\mathrm{NLR}$ & 0.034 & 0.048 \\
$\quad \mathrm{NLR}<2.2$ & $30(38.0 \%)$ & \\
$\mathrm{NLR} \geq 2.2$ & $49(62.0 \%)$ & & \\
$\mathrm{PLR}$ & & 0.118 & 0.149 \\
$\mathrm{PLR}<169.7$ & $54(68.4 \%)$ & & \\
$\mathrm{PLR} \geq 169.7$ & $25(31.6 \%)$ & & \\
$\mathrm{EOS}$ & & 0.001 & \\
$\mathrm{EOS}<0.1$ & $37(46.8 \%)$ & & \\
$\mathrm{EOS} \geq 0.1$ & $42(53.2 \%)$ & & \\
$\mathrm{PNI}$ & & 0.078 \\
$\mathrm{PNI}<55.9$ & $64(81.0 \%)$ & & \\
$\mathrm{PNI} \geq 55.9$ & $15(19.0 \%)$ & & \\
$\mathrm{Fbg}$ & & 0.100 \\
$\mathrm{Fbg}<345.4$ & $36(45.6 \%)$ & & \\
$\mathrm{Fbg} \geq 345.4$ & $43(54.4 \%)$ & & \\
\hline
\end{tabular}

NLR: neutrophil-lymphocyte ratio; PLR: platelet-lymphocyte ratio; EOS: eosinophilic granulocyte; PNI: prognostic nutrition index; Fbg: fibrinogen

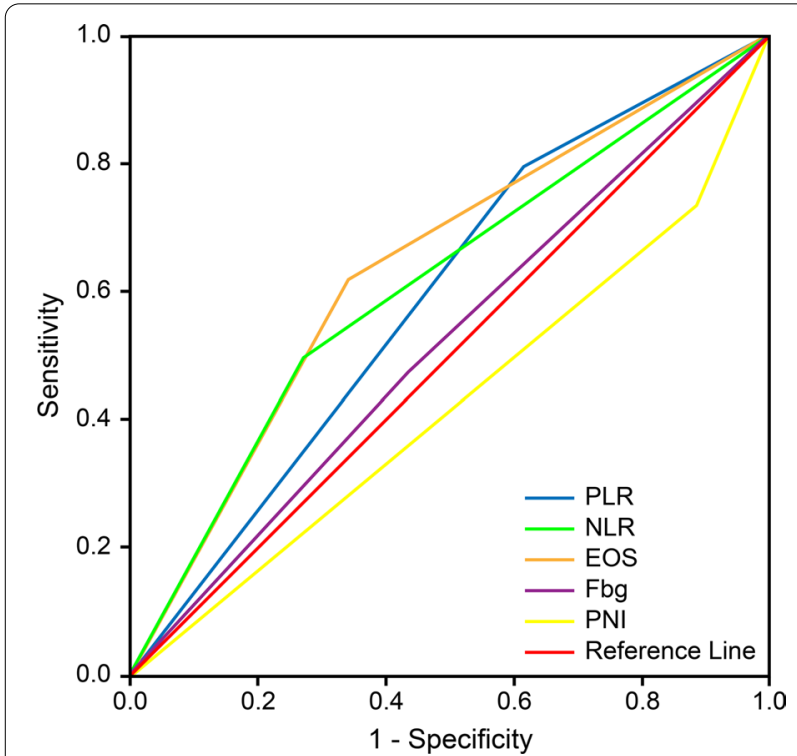

Fig. 2 ROC curves of inflammation-based and nutrition-related scores. The area under the ROC curve (AUC) indicated the prognostic value of relevant factors. The EOS (eosinophilic granulocyte) showed an AUC of $0.638(P=0.037)$

listed in Table 3. The cut-off value of EOS was regarded as a best predictive factor, with an area under the ROC curve (AUC) of $0.638(\mathrm{P}=0.037)$ (Fig. 2).

\section{Analysis of prognosis factors and prediction models}

We analysed all related factors possibly involved in patient prognosis. In univariate analysis, NLR $(\mathrm{P}=0.034)$, EOS $(\mathrm{P}=0.001)$, PNI $(\mathrm{P}=0.049)$, and postoperative pathologic stage $(\mathrm{P}<0.001)$ significantly influenced OS; NLR $(\mathrm{P}=0.048)$ and postoperative pathologic stage $(\mathrm{P}<0.001)$ significantly influenced PFS. Multivariate analysis showed that $\operatorname{EOS}(\mathrm{P}=0.024)$ and postoperative pathologic stage $(\mathrm{P}=0.020)$ were independent factors related to $O S$, and postoperative pathologic stage $(\mathrm{P}<0.001)$ significantly influenced PFS.

Some inflammation-based, nutrition-related factors as well as postoperative pathologic stage showed a significant influence on OS and PFS in univariate and multivariate analyses, we included as many related factors as possible and integrated them into prognostic nomograms to build prediction models with a high degree of fitness. Each factor was assigned points according to its coefficient. We established prediction models for OS and PFS, and the $\mathrm{C}$-index of each was 0.814 and 0.722 , respectively. Calibration curves were generated to validate consistency between the actual survival and the survival probability predicted by the nomograms (Fig. 3). The results indicated that the nomograms were well calibrated.

Since postoperative pathologic stage correlated significantly with both OS and PFS, we further explored the relationship between inflammation-based and nutrition-based factors and downstaging and found that the EOS value was related to postoperative pathologic stage $(\mathrm{P}=0.038)$.

Multivariate analysis also indicated postoperative pathologic stage to be an independent factor related to metastasis $(\mathrm{P}=0.004, \mathrm{HR}=0.150,95 \%$ CI $0.041-0.552)$. In further analysis comparing the effect of postoperative $\mathrm{T}$ stage and $\mathrm{N}$ stage on metastasis, we found that postoperative T0-T2 stage suggested a much lower risk of metastasis $(\mathrm{P}=0.011, \mathrm{HR}=0.119,95 \% \mathrm{CI} 0.023-0.610)$. Other factors did not have significant influence on recurrence in situ or distant metastasis in multivariate analysis.

\section{Discussion}

As multimodal therapy has been approved for the treatment of AEG, we evaluated the ability of neoadjuvant chemoradiotherapy to improve the surgical effect and prognosis of patients and screened prognostic factors potentially related to survival. We demonstrated that neoadjuvant chemoradiation contributed to a higher rate of complete resection and potentially improved survival. Additionally, we established prediction models using significant prognostic factors in our analysis.

Routine radical surgery, that is, complete removal of the primary tumour (R0 resection) with lymphadenectomy, 


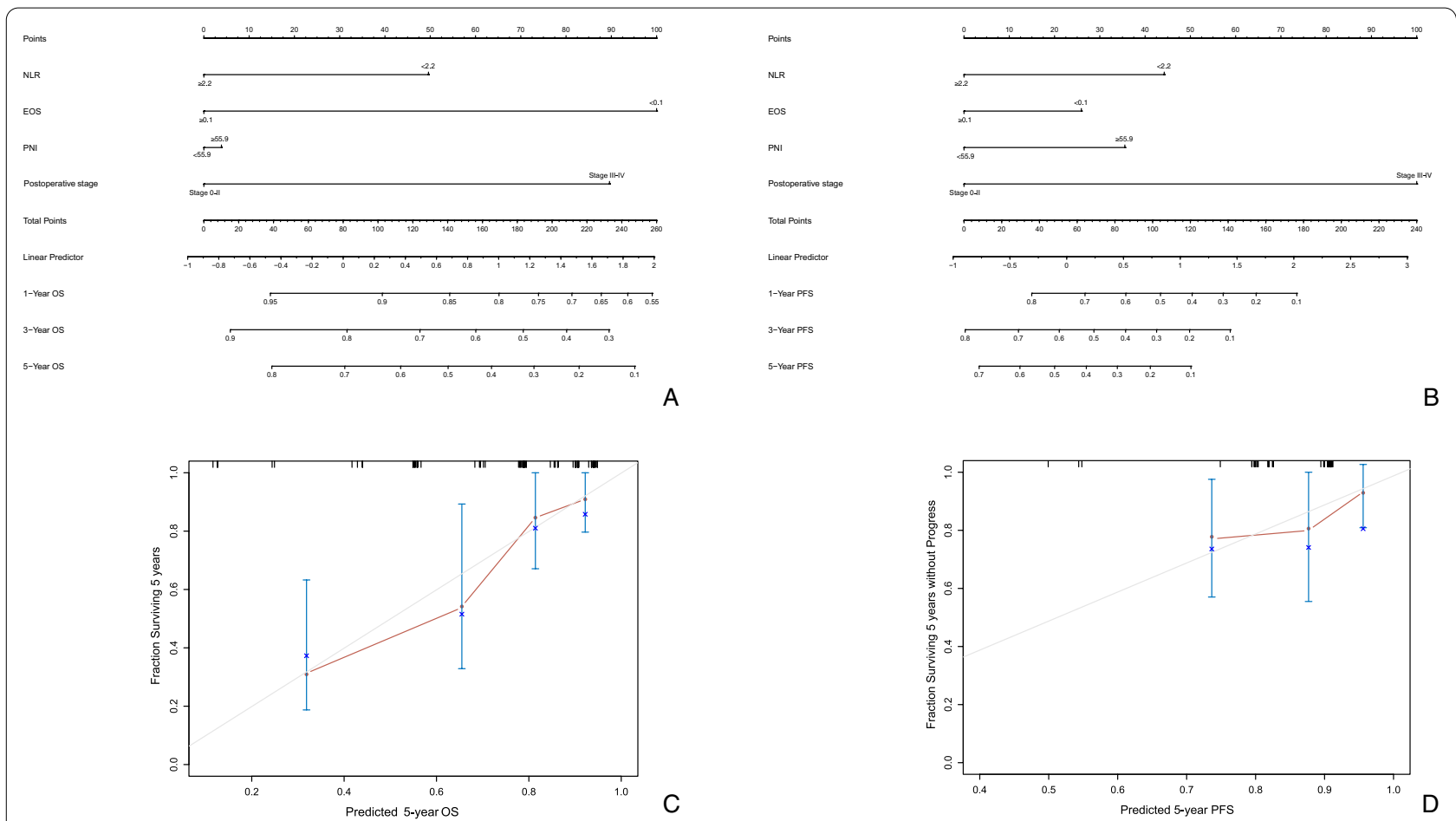

Fig. 3 Nomograms predicting OS (A) and PFS (B) rates of patients in our cohort. The nomogram adds up the points identified on the scale for each independent factor. The total scores projected on the bottom scale indicate the probabilities of 1-year, 3-year and 5-year OS rates and PFS rates. Calibration plots of the nomograms for 5-year OS prediction (C) and 5-year PFS prediction (D). The X axis displays the nomogram-predicted probability, and the $Y$ axis displays the actual survival rates estimated by the Kaplan-Meier method. The grey line represents excellent calibration, and the red line represents actual calibration. The blue vertical bars indicate $95 \% \mathrm{Cls}$

has been a mainstay for patients with resectable AEG. Multimodal therapy and complete resection without any residual disease enhance local control and even overall survival, and the quality of surgery is critical for both the short-term and long-term outcomes of patients with AEG $[24,25]$. Neoadjuvant therapy has been recommended, especially for patients whose clinical staging suggests a questionable chance of complete tumour removal by primary resection [26]. Total gastrectomy with a transhiatal resection of the distal oesophagus, combined with abdominal D2 lymphadenectomy, is suggested to be the best approach for Siewert II or III tumours [27-29], unless D2 surgery increases surgery-related risk [7].

We explored the effect of neoadjuvant chemoradiation on the results of surgery and prognosis of patients in this study. First, we found a high degree of neoadjuvant treatment completion, 74 of 79 patients completed the scheduled regimen of neoadjuvant therapy consecutively. There was no evidence indicating a remarkable increase in difficulty in the operation after chemoradiation or postoperative complications. There were no grade 4 toxic effects related to neoadjuvant treatment in our study; $78.5 \%$ of patients had grade 1 or 2 toxicities, indicating good tolerance to chemoradiation before surgery.
The postoperative mortality rate was $1.7 \%$, and the complications morbidity rate was $28.3 \%$, which compare favourably with the good results from western real-world clinical practice [30] and are comparable to the results for patients after radical surgery alone in our centre. Second, pathological analysis showed an ideal effect of neoadjuvant treatment, as R0 resection was achieved in $98.3 \%$ of patients, indicating a satisfactory effect of neoadjuvant treatment and high-quality surgery. Since the patients enrolled in our study had advanced primary tumours (T3-T4, mainly T4), suggesting a higher difficulty in achieving a complete response, the MPR rate of $46.7 \%$ and pathological downstaging rate of $91.7 \%$ still showed a favourable role of neoadjuvant chemoradiation in AEG treatment. A previous phase III trial used neoadjuvant chemotherapy combined with chemoradiation to achieve a pCR of approximately 15\% [1]; we achieved a similar effect without long-term induction chemotherapy. Our results also showed that neoadjuvant chemoradiotherapy reduced the lymph node metastasis rate by two-thirds; the number of positive lymph nodes in patients with lymphatic metastasis in our study was also extremely small. Overall, neoadjuvant chemoradiation may help to obtain a higher resection rate and pathologic response, as 
demonstrated by the postoperative nodal condition and TRG grade.

Overall survival in our study was comparable to that of previous studies: phase II/III trials and real-world studies have reported a 5-year OS rate of $45.0-65.1 \%$ $[1,11,31]$. Multivariate analysis showed that postoperative pathologic stage significantly influenced survival. In accordance with some previous studies [32,33], patients who remained at stage III-IV without downstaging after neoadjuvant treatment had poorer survival. The reason may be that the pathological status of primary tumours and metastatic lymph nodes represents a response to neoadjuvant treatment; patients with a poorer therapeutic response and poorer tumour necrosis might be resistant to the treatment, with a tendency to maintain a higher stage and more residual tumour than patients with a good response. It has been argued that the overall survival of patients whose disease does not respond to neoadjuvant therapy might even be worse than that of patients who accepted surgery alone because adequate surgery is substantially delayed $[34,35]$.

We generated prediction models for OS and PFS using nomograms and obtained a high $\mathrm{C}$-index for each model, indicating high predictive value. Calibration curves confirmed consistency between the predicted and actual trends, and the results showed that the nomograms were well calibrated. Overall, inflammation-based and nutrition-related factors and postoperative pathologic stage appear to contribute to establishing an applicable prediction model.

Among all factors included in the nomograms, we found some haematological indices to be consequential in predicting the prognosis of AEG carcinoma. Since the inflammation caused by tumours can lead to DNA damage, uncontrolled cell growth and micrometastasis, inflammation markers may be related to survival [36]. NLR and PLR are common inflammation-based factors for various digestive carcinomas [18], but the value of NLR and PLR in predicting prognosis was not unified in previous studies. NLR showed a significant influence in univariate analysis in this study and was included in the nomogram analysis. Yu et al. [16] recognized that serum fibrinogen correlates positively with advanced tumour stage and poor survival in gastric cancer and that the preoperative serum fibrinogen level might be an independent risk factor; however, this factor failed to show value in predicting AEG prognosis in our study. Preoperative nutritional status has been reported to be one of the critical factors for patient outcomes in a variety of surgeries, especially in gastrectomy. PNI is an important index evaluating both immune and nutrition status. Han et al. [37] suggested that AEG patients with a high PNI have a longer OS. The mechanism might be explained in two ways: first, a high level of PNI might indicated a good nutritional condition, resulting in better tolerance to treatment and better outcomes [17]; second, the lymphocyte count is part of the PNI score, and a low lymphocyte level might be associated with immunosuppression, which can lead to tumour progression or recurrence of residual tumours [38]. PNI functioned as a predictive factor in our models and should be evaluated in future studies. Notably, EOS is reported to be a possible predictor for the prognosis of colorectal cancers, whereby it is higher in colorectal carcinoma patients with better prognosis [15]. Studies on the value of EOS in other types of carcinomas are limited. Our study provides more evidence on the effect of EOS on patient survival, as our results showed that a higher level of EOS indicated better overall survival; it was also significantly related to postoperative stage. In summary, NLR $\geq 2.2$, EOS $\geq 0.1, \mathrm{PNI}<55.9$ and postoperative pathologic stage 0 -II might indicate positive OS and PFS outcomes. The prognostic factors above may all be regarded as valuable in prediction models, and how to apply them in screening patients with potential benefits needs to be confirmed in future studies.

There were some limitations in our study. The heterogeneity of retrospective studies is considered a major factor, as selection bias and recall bias are difficult to eliminate, and a prospective study is needed to verify our models. Additionally, our sample size limited the precision of analysis. Expanding the sample size and long-term follow-up are needed to obtain more specific outcomes and screen patients with potential benefits.

\section{Conclusions}

Our study showed a favourable downstaging and pathologic response after neoadjuvant chemoradiation in AEG patients, with acceptable adverse effects. Inflammation-based and nutrition-related factors, such as NLR, EOS, PNI, and postoperative pathologic stage, contribute to establishing an applicable prediction model of survival.

\footnotetext{
Abbreviations

AEG: Adenocarcinoma of the oesophagogastric junction; MPR: Major pathologic response; OS: Overall survival; PFS: Progression-free survival; NLR: Neutrophil-lymphocyte ratio; PNI: Prognostic nutrition index; EOS: Eosinophilic granulocyte; EGJ: Oesophagogastric junction; Fbg: Fibrinogen; ECOG: Eastern Cooperative Oncology Group; CT: Computer tomography; VMAT: Volumetricmodulated Arc Therapy; CBCT: Cone beam computer tomography; GTV: Gross tumour volume; CTV: Clinical tumour volume; SD: Stable disease; PR: Partial response; CR: Complete response; TRG: Tumour regression grade; AUC: Area under the ROC curve.
}

Acknowledgements Not applicable. 


\section{Authors' contributions}

RX Du, J Ming and JH Geng contributed equally in collecting and analyzing the data, and were main writers of this manuscript. YH Li, ZY Li and WH Wang made equal contributions in designing and organizing this study. XG Zhu, YZ Zhang, S Li, ZY Liu and HZ Wang made contributions in discussion part and helped with proofreading. ZL Wang, L Tang provided medical imaging data and analyses. XT Zhang, AW Wu, ZD Bu, Y Yan and ZW Li provided clinical data, pathological data and analyses. All authors read and approved the final manuscript.

\section{Funding}

Supported by the programs below in collecting data and writing the manuscript: (1) Capital's Funds for Health Improvement and Research No. 2020-2-1027; (2) Beijing Municipal Science \& Technology Commission No. Z181100001718192; (3) National Natural Science Foundation No. 82073333.

\section{Availability of data and materials}

All data generated or analyzed during this study are included in this published article and its supplementary information files.

\section{Declarations}

Ethics approval and consent to participate

Our study was approved by Medical Ethic Committee of Peking University Cancer Hospital and Institute (the number of ethic approval: 2014KT74).

\section{Consent for publication}

Not applicable.

\section{Competing interests}

The authors declare that they have no competing interests.

\section{Author details}

${ }^{1}$ Key Laboratory of Carcinogenesis and Translational Research (Ministry of Education/Beijing), Department of Radiation Oncology, Peking University Cancer Hospital and Institute, Beijing 100142, People's Republic of China. ${ }^{2}$ Key Laboratory of Carcinogenesis and Translational Research (Ministry of Education/Beijing), Department of Medical Imaging, Peking University Cancer Hospital and Institute, Beijing 100142, People's Republic of China. ${ }^{3}$ Key Laboratory of Carcinogenesis and Translational Research (Ministry of Education/Beijing), Department of Gastrointestinal Oncology, Peking University Cancer Hospital and Institute, Beijing 100142, People's Republic of China. ${ }^{4}$ Key Laboratory of Carcinogenesis and Translational Research (Ministry of Education/Beijing), Department of Gastrointestinal Surgery, Peking University Cancer Hospital and Institute, Beijing 100142, People's Republic of China. ${ }^{5}$ Key Laboratory of Carcinogenesis and Translational Research (Ministry of Education/ Beijing), Endoscopy Center, Peking University Cancer Hospital and Institute, Beijing 100142, People's Republic of China. 'Key Laboratory of Carcinogenesis and Translational Research (Ministry of Education/Beijing), Department of Pathology, Peking University Cancer Hospital and Institute, Beijing 100142, People's Republic of China.

Received: 13 January 2022 Accepted: 17 February 2022 Published online: 03 March 2022

\section{References}

1. Stahl M, Walz MK, Stuschke M, Lehmann N, Meyer HJ, Riera-Knorrenschild J, et al. Phase III comparison of preoperative chemotherapy compared with chemoradiotherapy in patients with locally advanced adenocarcinoma of the esophagogastric junction. J Clin Oncol. 2009;27:851-6.

2. Arnold M, Soerjomataram I, Ferlay J, Forman D. Global incidence of oesophageal cancer by histological subtype in 2012. Gut. 2015;64:381-7.

3. Demeester SR. Adenocarcinoma of the esophagus and cardia: a review of the disease and its treatment. Ann Surg Oncol. 2006;13:12-30.

4. Siegel R, Ma J, Zou Z, Jemal A. Cancer statistics, 2014. CA Cancer J Clin. 2014;64:9-29.
5. Sung J, Ng E, Lin J, Ho K, Ji J, Sugano K, et al. Digestive cancer management in Asia: position statements: a report on Gl Oncology Summit in 2011. J Gastroenterol Hepatol. 2012;27:1417-22.

6. Hong Y, Wu AW, Li ZY, Bu ZD, Ji JF. Surgical treatment results and prognostic analysis of 514 cases with gastroesophageal junction carcinoma. Zhonghua Wai Ke Za Zhi. 2010;48:1289-94.

7. Hasegawa S, Yoshikawa T. Adenocarcinoma of the esophagogastric junction: incidence, characteristics, and treatment strategies. Gastric Cancer. 2010;13:63-73.

8. Sasako M, Sano T, Yamamoto S, Sairenji M, Arai K. Left thoracoabdominal approach versus abdominal-transhiatal approach for gastric cancer of the cardia or subcardia: a randomised controlled trial. Lancet Oncol. 2006;7:644-51.

9. Van HP, Hulshof MC, Lanschot JV, Steyerberg EW, Van MI, Wijnhoven $\mathrm{BP}$, et al. Preoperative chemoradiotherapy for esophageal or junctional cancer. J Med Imaging Radiat Oncol. 2012;43:215-9.

10. Zhang Z, Gu X, Yin W. Value of Preoperative radiotherapy for adenocarcinoma gastric cardia (AGC): a prospective randomized trial on 370 patients. Chin J Of radiat Oncol. 1998;

11. Yang W, Zhou M, Li G, Shen L, Zhang Z. Preoperative chemoradiotherapy versus postoperative chemoradiotherapy for patients with locally advanced gastric cancer: a retrospective study based on propensity score analyses. Front Oncol. 2020;10:560115.

12. Fiorica F, Bona DD, Schepis F, Licata A, Shahied L, Venturi A, et al. Preoperative chemoradiotherapy for oesophageal cancer: a systematic review and meta-analysis. Gut. 2004;53:925-30.

13. Urschel JD, Vasan $\mathrm{H}$, Blewett CJ. A meta-analysis of randomized controlled trials that compared neoadjuvant chemoradiation and surgery to surgery alone for resectable esophageal cancer. Am J Surg. 2003;183:274-9.

14. Kudou K, Nakashima Y, Haruta Y, Nambara S, Mori M. Comparison of inflammation-based prognostic scores associated with the prognostic impact of adenocarcinoma of esophagogastric junction and upper gastric cancer. Ann Surg Oncol. 2020;28:2059-67.

15. Korneva YS, Ukrainets RV, Dorosevich AE. Eosinophilic granulocyte in peripheral blood as biological marker for colon cancer prognosis. Biol Markers Fundam Clin Med (Collect Abstr). 2020;4:4-5.

16. Yu X, Hu F, Yao Q, Li C, Zhang H, Xue Y. Serum fibrinogen levels are positively correlated with advanced tumor stage and poor survival in patients with gastric cancer undergoing gastrectomy: a large cohort retrospective study. BMC Cancer. 2016;16:480.

17. Wen J, Bedford M, Begum R, Mitchell H, Hodson J, Whiting J, et al. The value of inflammation based prognostic scores in patients undergoing surgical resection for oesophageal and gastric carcinoma. J Surg Oncol. 2018;117:1697-707

18. Zhang L, Su Y, Chen Z, Wei Z, Han W, Xu A. The prognostic value of preoperative inflammation-based prognostic scores and nutritional status for overall survival in resected patients with nonmetastatic Siewert type II/III adenocarcinoma of esophagogastric junction. Medicine. 2017;96:e7647.

19. Siewert JR, Stein HJ. Carcinoma of the cardia: carcinoma of the gastroesophageal junction-classification, pathology and extent of resection. Dis Esophagus. 1996;9:173-82.

20. Hirahara N, Tajima Y, Fujii Y, Kaji S, Yamamoto T, Hyakudomi R, et al. Prognostic nutritional index as a predictor of survival in resectable gastric cancer patients with normal preoperative serum carcinoembryonic antigen levels: a propensity score matching analysis. BMC Cancer. 2018;18:285.

21. Camp RL, Dolled-Filhart M, Rimm DL. X-tile: a new bio-informatics tool for biomarker assessment and outcome-based cut-point optimization. Clin Cancer Res. 2004;10:7252-9.

22. Eisenhauer EA, Therasse P, Bogaerts J, Schwartz LH, Sargent D, Ford R, et al. New response evaluation criteria in solid tumours: revised RECIST guideline (version 1.1). Eur J Cancer. 2016;45:228-47.

23. Díaz-González JA, Rodríguez J, Hernández-Lizoain JL, Ciérvide R, Gaztañaga M, San Miguel I, et al. Patterns of response after preoperative treatment in gastric cancer. Int J Radiat Oncol Biol Phys. 2011;80:698-704.

24. Siewert JR, Feith M, Stein HJ. Biologic and clinical variations of adenocarcinoma at the esophago-gastric junction: relevance of a topographicanatomic subclassification. J Surg Oncol. 2010;90:139-46.

25. Kappas AM, Roukos DH. Quality of surgery determinant for the outcome of patient with gastric cancer. Ann Surg Oncol. 2002;9:828-30.

26. Stein HJ, Feith M, Siewert JR. Cancer of the esophagogastric junction. Surg Oncol. 2000;9:35-41. 
27. Kakeji Y, Yamamoto M, Ito S, Sugiyama M, Egashira A, Saeki H, et al. Lymph node metastasis from cancer of the esophagogastric junction, and determination of the appropriate nodal dissection. Surg Today. 2012;42:351-8.

28. Siewert JR, Ott K. Are squamous and adenocarcinomas of the esophagus the same disease? Semin Radiat Oncol. 2007;17:38-44.

29. Siewert J, Feith M, Werner M, Stein H, Wong J. Adenocarcinoma of the esophagogastric junction: results of surgical therapy based on anatomical/topographic classification in 1,002 consecutive patients. Ann Surg. 2000:232:353-61.

30. Carboni F, Lorusso R, Santoro R, Lepiane P, Mancini P, Sperduti I, et al. Adenocarcinoma of the esophagogastric junction: the role of abdominaltranshiatal resection. Ann Surg Oncol. 2009;16:304-10.

31. Burmeister BH, Thomas JM, Burmeister EA, Walpole ET, Harvey JA, Thomson $\mathrm{DB}$, et al. Is concurrent radiation therapy required in patients receiving preoperative chemotherapy for adenocarcinoma of the oesophagus? A randomised phase II trial. Eur J Cancer. 2011:47:354-60.

32. Chirieac LR, Swisher SG, Ajani JA, Komaki RR, Correa AM, Morris JS, et al. Posttherapy pathologic stage predicts survival in patients with esophageal carcinoma receiving preoperative chemoradiation. Cancer. 2005:103:1347-55.

33. Schröder W, Baldus SE, Mönig SP, Beckurts T, Dienes HP, Hölscher AH. Lymph node staging of esophageal squamous cell carcinoma in patients with and without neoadjuvant radiochemotherapy: histomorphologic analysis. World J Surg. 2002;26:584-7.

34. Hartgrink HH, Velde C, Putter H, Songun I, Tesselaar M, Kranenbarg EK, et al. Neo-adjuvant chemotherapy for operable gastric cancer: long term results of the Dutch randomised FAMTX trial. Eur J Surg Onco. 2004;30:643-9.

35. Kelsen DP, Ginsberg R, Pajak TF, Sheahan DG, Gunderson L, Mortimer J, et al. Chemotherapy followed by surgery compared with surgery alone for localized esophageal cancer. N Engl J Med. 1998:339:1979-84.

36. Balkwill F, Mantovani A. Inflammation and cancer: back to Virchow? Lancet. 2001:357:539-45.

37. Han WX, Chen ZM, Wei ZJ, Xu AM. Preoperative pre-albumin predicts prognosis of patients after gastrectomy for adenocarcinoma of esophagogastric junction. World J Surg Oncol. 2016;14:279.

38. Migita K, Takayama T, Saeki K, Matsumoto S, Wakatsuki K, Enomoto K, et al. The prognostic nutritional index predicts long-term outcomes of gastric cancer patients independent of tumor stage. Ann Surg Oncol. 2013:20:2647-54.

\section{Publisher's Note}

Springer Nature remains neutral with regard to jurisdictional claims in published maps and institutional affiliations.

Ready to submit your research? Choose BMC and benefit from:

- fast, convenient online submission

- thorough peer review by experienced researchers in your field

- rapid publication on acceptance

- support for research data, including large and complex data types

- gold Open Access which fosters wider collaboration and increased citations

- maximum visibility for your research: over 100M website views per year

At BMC, research is always in progress.

Learn more biomedcentral.com/submissions 